ISSN 1997-5902

\title{
Étude floristique et ethnobotanique de la flore médicinale du Haut Atlas oriental (Haute Moulouya)
}

\author{
Noureddine Benlamdini, Mohamed Elhafian, Atmane Rochdi \& Lahcen Zidane \\ Laboratoire de Biodiversité et Ressources Naturelles, Université Ibn Tofaill, Faculté des Sciences, BP 133, Kénitra, \\ Maroc. \\ Auteur de la correspondance : nourdben@gmail.com.
}

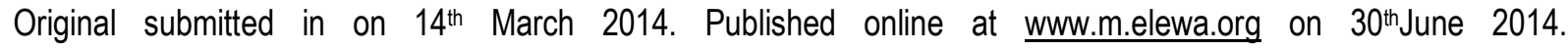
http://dx.doi.org/10.4314/iab.v78i1.17

\section{RESUME :}

Objectif: La présente étude est une contribution à la connaissance des plantes médicinales utilisées en phytothérapie traditionnelle par la population locale de la Haute Moulouya.

Méthodologie et résultats : A l'aide de 320 fiches questionnaires, une série d'enquêtes ethnobotaniques a été menée sur le terrain durant les années 2012 et 2013. La localisation des différents milieux, d'enquêtes ethnobotaniques et de relevés floristiques, a été repérée par la méthode d'échantillonnage stratifié et aléatoire. L'étude de la flore médicinale a fait ressortir une richesse floristique de 115 espèces médicinales réparties en 45 familles et 99 genres avec une prédominance des familles suivantes : Lamiaceae (20), Asteraceae (11), Apiaceae (8), Fabaceae (7), Rosaceae (6), et Poaceae (5). Les résultats de cette étude ont montré aussi que le feuillage constitue la partie la plus utilisée, et la majorité des remèdes est préparée sous forme de décoction. Les plantes médicinales sont souvent utilisées pour soigner les affections digestives (44\%), et $40 \%$ de ces plantes ont provoqué des intoxications.

Conclusion et application de la recherche : Les enquêtes effectués ont permis de recueillir les informations sur les plantes médicinales (les données floristiques, la partie utilisée, le mode de préparation et la toxicité) et sur le profil de l'informateur (sexe, âge, le niveau d'instruction) et ont permis également d'évaluer l'impact de la collecte des plantes médicinales sur l'environnement. Par ailleurs, cette étude pourrait avoir une grande valeur bibliographique pour les recherches dans les domaines de la phytochimie et de la pharmacologie et une assise de base pour les générations futures.

Mot clés : Plantes médicinales, Ethnobotanique, floristique, phytothérapie, Haute Moulouya.

\begin{abstract}
Objective: This study is considered as a contribution to the study of medicinal plants that are used in traditional herbal medicne by the local population of the Haute Moulouya.

Methods and results: Using 320 questionnaires, a series of ethnobotanical surveys was conducted in the field during the years of 2012 and 2013. The location of different backgrounds for the floristic and ethnobotanical surveys was spotted by the stratified random sampling method. The study of medicinal plants showed a floristic richness of 115 medicinal species divided into 45 families and 99 genera with a predominance of the following families: Lamiaceae (20), Asteraceae (11), Apiaceae (8), Fabaceae (7) Rosacea (5), and Poaceae (5). The results of this study also showed that the foliage is the most used part, and the majority of remedies are
\end{abstract}


prepared as a decoction. Herbal remedies are often used to treat digestive disorders (44\%), and $40 \%$ of these plants have caused poisoning.

Conclusion and Application: These surveys provide information on medicinal plants (floristic data, the used part, mode of preparation, and toxicity) and the profile of the informant (gender, age, level of education,...). They have also assessed the impact of the collection of medicinal plants on the environment. Moreover, this study could offer an important value for bibliographic researches in the fields of phytochemistry, pharmacology and a seat base for future generations.

Keywords: medicinal plants, ethnobotany, floristic, traditional herbal medicine, Haute Moulouya.

\section{NTRODUCTION}

Dans sa quête de nourriture, l'homme primitif a fait l'essai de toutes sortes de plantes. Certaines l'ont nourri, d'autres l'ont guéri de ses maux, quelquesunes ont entraîné sa mort (Evans Schultes, 1982). L'évolution de la connaissance des espèces médicinales par les tests de longues dates s'est concrétisée chez les anciens herboristes par l'établissement de listes d'espèces et de formules de remèdes à base de plantes. Aujourd'hui, ce savoirfaire traditionnel constitue d'une part un trésor d'informations pour ceux et celles qui préfèrent les usages populaires pour aborder leurs maux quotidiens et d'autre part une ressource inestimable pour l'industrie pharmaceutique (Guedje et al., 2010). Étant donné que $50 \%$ des produits pharmaceutiques disponibles sur le marché sont d'origine naturelle (Hamilton, 2003). En Afrique, Les plantes médicinales constituent des ressources précieuses pour la majorité des populations rurales, où plus de $80 \%$ de cette population s'en sert pour assurer leurs soins de santé (Jiofack et al., 2010 ; Mpondo et al., 2012). De même, le continent africain regorge de très grande diversité d'espèces végétales, dont la majorité est utilisée comme médicaments. En effet, sur les 300000 espèces végétales recensées sur la planète, plus de 200000 espèces vivent dans les pays tropicaux d'Afrique et ont des vertus médicinales (Sofowora, 1993). Le Maroc, de par sa situation géographique, constitue un cadre naturel tout à fait original, offrant une gamme complète de bioclimats méditerranéens favorisant une flore riche et variée avec un endémisme très marqué (Ghanmi et al., 2011). Il est l'un des pays méditerranéens qui ont une longue tradition médicale et un savoir-faire traditionnel à base de plantes médicinales (Scherrer et al., 2005). En effet, sur les 5000 espèces et sous-espèces répertoriées en Afrique du nord, 4200 poussent au Maroc, dont au moins 500 sont potentiellement aromatiques et/ou médicinales et 250 sont utilisées (Sijelmassi, 1991). Néanmoins, les espèces naturelles au Maroc connaissent actuellement une dégradation très intense et très poussée ; à cause de l'exploitation intensive de ces espèces pour des besoins médicaux. En effet, la quasi-totalité des espèces médicinales utilisées au Maroc proviennent de sources sauvages, les cultures de plantes médicinales demeurant relativement limitées, c'est ainsi que, l'exploitation de ces espèces au Maroc est loin d'être rationnelle. Devant cette situation alarmante, il est rendu de plus en plus difficile à protéger et sauvegarder ces écosystèmes. En outre, l'analyse de la bibliographie médicinale marocaine montre que les données relatives aux plantes médicinales régionales sont très fragmentaires et dispersées (Bammi et Douira, 2002 ; Benkhnigue et al., 2010-2011). De même, le savoir populaire est détenu actuellement par peu de personnes parmi lesquelles on trouve un taux d'analphabétisme élevé (Hseini et Kahouadji, 2007). Ainsi, il est devenu nécessaire de sauvegarder et de traduire ce savoirfaire populaire en savoir scientifique afin de l'utiliser d'une manière rationnelle. C'est dans cette optique qu'une étude floristique et ethnobotanique a été menée dans la région de la Haute Moulouya qui présente une diversité écologique, lithologique et floristique assez importante. Cette étude consiste en une série d'enquêtes ethnobotanique en vue de recenser les plantes utilisées en pharmacopée traditionnelle au niveau de cette région, de collecter le maximum d'informations sur les modalités d'utilisation et d'exploitation de ces plantes dans la médecine traditionnelle. 


\section{PRESENTATION DE LA ZONE D'ETUDE}

La zone d'étude, la Haute Moulouya, fait partie administrativement du cercle de Midelt (région de Meknès-Tafilalet) et hydrauliquement du bassin versant de la Moulouya. Elle est délimitée au Nord par la province d'Ifrane, à l'Est par la province de Boulmane et ErRachidia, au Sud par la province d'Er-Rachidia, et à l'Ouest par l'annexe de Boumia et Lakbab. Cette région est située à $250 \mathrm{~km}$ de l'Océan Atlantique, et isolée par $150 \mathrm{~km}$ de chaînes de montagne (Rhanem, 2009). Elle est enclavée entre les chaînes montagneuses du moyen et haut Atlas, de position géographique caractérisée par un relief très accidenté et d'altitude variant de 1400 à $1800 \mathrm{~m}$ au-dessus de la mer. Le point culminant est le Jbel El Ayachi avec $3750 \mathrm{~m}$ (Fig.1). L'aire géographique de cette région couvre une superficie de $329.000 \mathrm{Ha}$ dont $45.00 \mathrm{Ha}$ de superficie agricole (27.830 Ha Bour, 17.170 $\mathrm{Ha}$ Irrigués) et constituée de $22 \%$ de zones montagneuses, $71 \%$ de collines et plateaux et $7 \%$ de plaines localisées dans les périmètres Outat, Ansegmir, Amersid et Itzer (Monographie provinciale, 2009). Cette cuvette a connu au cours du Jurassique une évolution géodynamique conditionnée par la dislocation de la plate- forme carbonatée liasique qui est responsable de la structuration de cette région en blocs basculés, organisés en une mosaïque de petits bassins (dépocentres) que séparent des zones hautes (Igmoullan et al., 2001). Les sols présentent fréquemment à faible profondeur un horizon riche en calcaire souvent durci et feuilleté. Ces croûtes calcaires constituent un facteur très défavorable à la végétation, car elles empêchent l'infiltration des eaux et la pénétration des racines en profondeur (Emberger et Lemée, 1962; El Hai, 1968; Rhanem, 2009). La température moyenne annuelle est de $14,3^{\circ} \mathrm{C}$, avec une moyenne des minima journaliers de $7,6^{\circ} \mathrm{C}$ et une moyenne des maxima journaliers de $21,0^{\circ} \mathrm{C}$ (Rhanem, 2009). Le climatogramme d'Amberger a déterminé trois étages bioclimatiques (DPAPK, 2005): l'étage présaharien limitrophe des provinces de Sud (ErrachidiaMissour), caractérisé par une pluviométrie faible inférieure à $100 \mathrm{~mm} /$ an et un été chaud et sec ; l'étage aride rencontré généralement à Midelt et Aït lzdeg et dont la pluviométrie moyenne annuelle n'excède pas 200 $\mathrm{mm}$; et l'étage semi- aride marqué nettement dans les communes rurales Ait Ayach et Itzer avec une pluviométrie moyenne annuelle supérieure à $200 \mathrm{~mm}$.

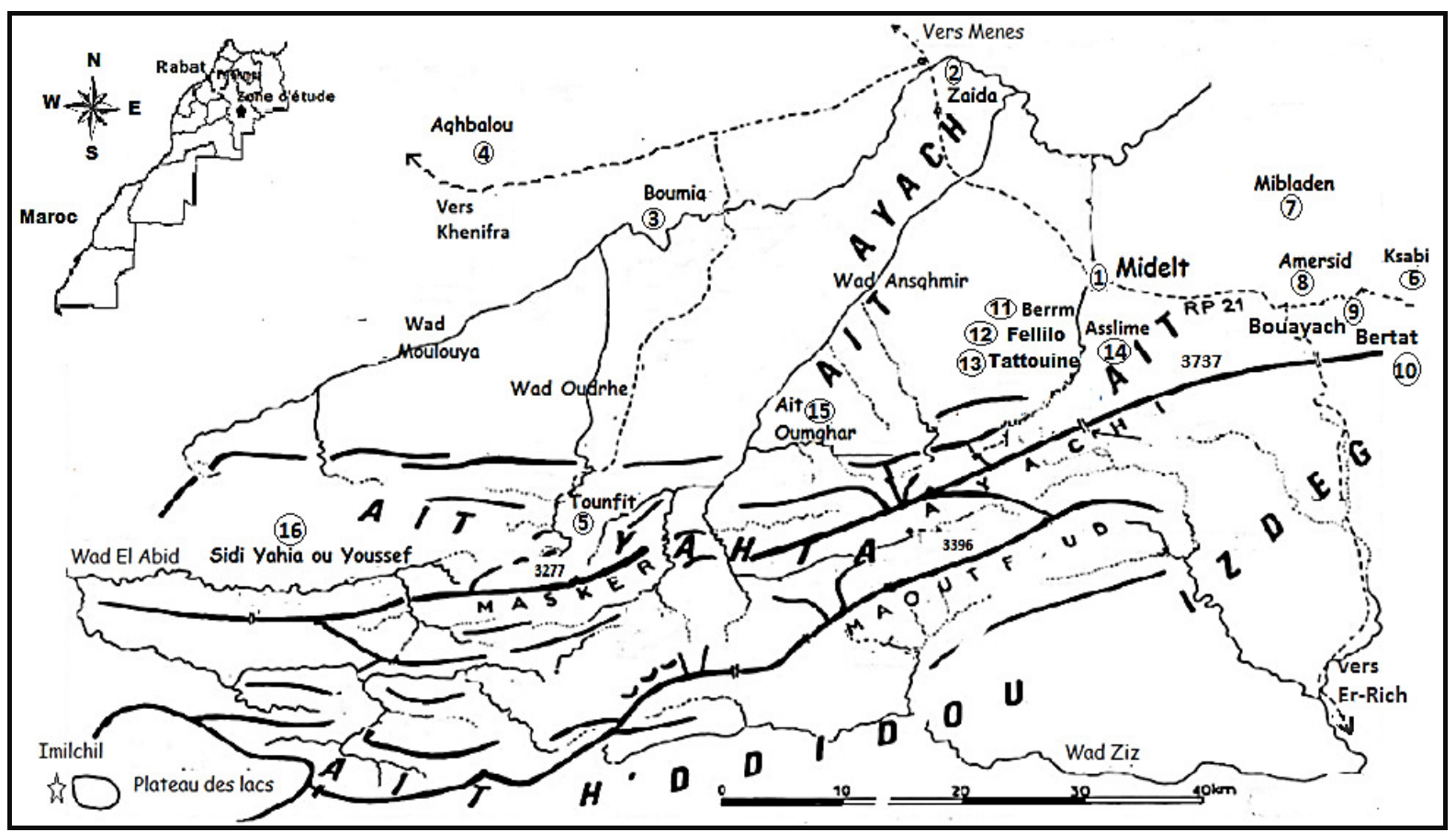

Fig.1. Carte schématique de la zone d'étude- carte modifiée- (Peyron, 1976). 
La zone de la Haute Moulouya comporte plusieurs systèmes de culture dont les principales composantes sont (DPAPK, 2005): la céréaliculture $(16421 \mathrm{Ha})$, l'arboriculture $(4.509,50 \mathrm{Ha}$ dont le pommier : $3.420 \mathrm{Ha})$, cultures fourragères $(1.742 \mathrm{Ha})$, les cultures maraîchères $(1.444,50 \mathrm{Ha})$, et les légumineuses $(520 \mathrm{Ha})$. Hydrologiquement, les principales rivières du Haut Atlas

\section{METHODE D'ETUDE}

Notre étude sur le terrain a été effectuée pendant les années 2012 et 2013 ; période au cours de laquelle nous avons réalisé des entrevues ethnobotaniques auprès de la population locale de la Haute Moulouya. Dans un premier temps, nous avons cherché à repérer les différents milieux d'enquêtes par la méthode d'échantillonnage stratifié (Kahouadji, 1986). Cette technique a pour but d'avoir un inventaire floristique le plus complet possible et de réaliser des enquêtes ethnobotaniques variées d'une zone à une autre dans la région étudiée (Lahsissene et al., 2009). Par conséquent, la dition a été divisée en 16 strates selon les descripteurs du milieu notamment le climat, le sol et la végétation (Fig.1). En procédant par un échantillonnage aléatoire stratifié, des échantillons de 20 personnes sont enquêtées dans chacune des 16 strates, dont six communes urbaines ( 1 :Midelt ; 2 : Zaida ; 3 : Boumia ; $4:$ Aghbalou; 5 : Tounfit; $6:$ Ksabi) et dix communes rurales (7 :Mibladen ; 8 :Amersid ; 9 :Bou Ayache ; 10 : Bertat; 11: Berrm; 12: Fellilo; 13: Tattouine; 14 : Asslime ; 15 : Ait oumghar ; 16 : Sidi yahia ou youssef) et ils sont mis ensemble pour constituer l'échantillon global de 320 personnes (Fig. 1). Les enquêtes ethnobotaniques

\section{RESULTATS ET DISCUSSION PLACE DE LA PHYTOTHÉRAPIE DANS LE SYSTĖME DE SOIN}

Choix entre la phytothérapie et la médecine moderne: L'enquête menée auprès de la population locale de la Haute Moulouya a révélé que selon les systèmes de soin, la population peut être répartie en 3 classes (Tableau1). Sur 320 personnes interrogées, on distingue :

- 69 personnes ont recours à la phytothérapie seule ;

- 64 personnes ont recours à la médecine moderne seule ;

- 187 personnes ont recours à la phytothérapie et à la médecine moderne à la fois.

Les personnes qui pratiquent la phytothérapie sont donc en nombre de 256 (80\%) dont la plupart sont des calcaire alimentant la Moulouya sont d'W en E : l'oued Oudhres, puis l'Ansegmir et l'oued Outat; ce sont les seules qui soient pérennes (Fig.1). Ces principaux affluents de la Moulouya originaires du haut atlas présentent des débits soutenus grâce aux sources issues des puissantes formations carbonatées (El Gasmi et al., 2013).

ont été réalisées à l'aide de 320 fiches questionnaires. Lors de chaque entretien nous avons collecté toutes les informations sur l'informateur et les plantes médicinales utilisées par celui-ci. Ainsi, en plus du profil de chaque informateur (âge, sexe, niveau d'instruction, situation familiale, et lieu de résidence). Les renseignements recueillis pour chaque plante comprennent le nom vernaculaire, le type de maladies traitées, la partie utilisée et le mode de préparation. L'identification taxonomique des espèces récoltées lors des relevés floristiques, a été faite ultérieurement au laboratoire de Biodiversité et Ressources Naturelles de Facultédes sciences de Kénitra, en utilisant des ouvrages botaniques tels que: "Flore pratique du Maroc. Manuel de détermination des plantes Vol 1 et $2 »$ de Fennane et al. (1999 et 2007), «La pharmacopée marocaine traditionnelle, médecine arabe ancienne et savoir populaire» de Bellakhdar (1997) et "Les plantes médicinales du Maroc» de Sijelmassi (1993). Les informations ethnobotaniques rassemblées lors des enquêtes réalisées sur le terrain ont été saisies dans une base de données puis traitées par l'outil «EXCEL».

villageois. Le choix de la phytothérapie par la population locale est relié le plus souvent à l'enclavement des zones rurales, l'inexistence ou l'état rudimentaire des infrastructures sanitaires, le coût élevé des préparations pharmaceutiques, ainsi que la modicité des revenus des populations (Guedje et al., 2010).

Utilisation des plantes médicinales selon l'âge : La moyenne d'âge de notre dition est d'environ 48 ans avec des extrêmes d'âge allant de 15 à 85 ans. L'utilisation des plantes médicinales dans la zone de la Haute Moulouya est répandue chez toutes les tranches d'âge avec prédominance des personnes d'âge supérieur à 60 ans $(26 \%)$. Les tranches d'âge, de 31 à 40 ans, 41 à 50 ans et 51 à 60 ans, viennent ensuite avec respectivement $22 \%, 18 \%$ et $17,51 \%$. Cependant les personnes de tranche d'âge de 21 ans à 30 ans $(10,77 \%)$ et d'âge $\leq$ 20 ans $(5.72 \%)$ ne recourent pas beaucoup à la médecine 
traditionnelle pour leur sécurité médicale (Tableau 1). La connaissance des usages des plantes médicinales et leurs propriétés, est généralement acquise suite à une longue expérience accumulée et transmise d'une génération à l'autre. La transmission de cette connaissance est actuellement en danger parce qu'elle n'est pas toujours assurée (Anyinam 1995 ; Mehdioui et Kahouadji, 2007 ; Benkhnigue, 2010). Ces résultats confirment effectivement que les personnes âgées connaissent bien la phytothérapie traditionnelle par rapport aux autres tranches d'âges. De même, le faible intérêt pour les effets thérapeutique des plantes marqué chez les personnes de tranche d'âge de 21 à 30 ans s'explique par la méfiance particulièrement des jeunes qui ont tendance à ne plus trop croire en cette médecine traditionnelle (Mehdioui et Kahouadji, 2007).

Utilisation des plantes médicinales selon le sexe : A l'échelle de la zone d'étude les femmes et les hommes sont concernés par la médecine traditionnelle, avec une légère prédominance des femmes. En effet, $90,27 \%$ des femmes enquêtées utilisent des plantes médicinales contre $88,35 \%$ des hommes. De plus, parmi les individus qui pratiquent la médecine traditionnelle, $53 \%$ sont des femmes et $47 \%$ sont des hommes (Tableau 1). Ceci peut être expliqué par le fait que les femmes sont traditionnellement les dépositaires des secrets des plantes médicinales. Héritières d'un riche savoir familial, par la transmission des connaissances, elles témoignaient avant tout, d'un savoir adapté à leur famille et à leurs besoins (Aquaron, 2005). Ces résultats sont conformes à ceux obtenus par Benkhnigue (2010) dans la région de Mechraâ Bel Ksiri et par Mehdioui et Kahouadji (2007) dans la forêt d'Amsittène (Province d'Essaouira).

Tableau1 : Profil général des enquêtés ( $\mathrm{N}=320$ personnes).

\begin{tabular}{|c|c|}
\hline Caractéristiques & Pourcentage \\
\hline $\begin{array}{l}\text { Prévalence : } \\
\text { - } \quad \text { Utilisateurs de la médecine traditionnelle. } \\
\text { - } \quad \text { Utilisateurs de la médecine moderne. } \\
\text { - } \quad \text { Utilisateurs de la médecine traditionnelle et moderne. }\end{array}$ & $\begin{array}{ll}\text { - } & 21,5 \% \\
\text { - } & 20 \% \\
\text { - } & 58,44 \%\end{array}$ \\
\hline $\begin{array}{ll}\text { Age : } & \\
- & \leq 20 \text { ans } \\
- & 21 \text { à } 30 \text { ans } \\
- & 31 \text { à } 40 \text { ans } \\
- & 41 \text { à } 50 \text { ans } \\
- & 51 \text { à } 60 \text { ans } \\
- & \geq 60 \text { ans }\end{array}$ & $\begin{array}{ll}\text { - } & 5,72 \% \\
\text { - } & 10,77 \% \\
\text { - } & 22 \% \\
\text { - } & 18 \% \\
\text { - } & 17,51 \% \\
\text { - } & 26 \% \\
\end{array}$ \\
\hline $\begin{array}{ll}\text { Sexe : } & \\
\bullet & \text { Femme } \\
- & \text { Homme } \\
\end{array}$ & $\begin{array}{ll}\text { - } & 53 \% \\
\text { - } & 47 \% \\
\end{array}$ \\
\hline \begin{tabular}{ll}
\multicolumn{2}{l}{ Niveau d'instruction : } \\
- & Analphabète \\
- & Primaire \\
- & Secondaire \\
- & Universitaire
\end{tabular} & $\begin{array}{ll}- & 41 \% \\
- & 26 \% \\
- & 24 \% \\
& 9 \%\end{array}$ \\
\hline
\end{tabular}

Utilisation des plantes médicinales selon le niveau d'instruction : Sur la totalité des usagers de la médecine traditionnelle les analphabètes dominent avec un pourcentage de $41 \%$ (Tableau1). Toutefois, les personnes ayant un niveau d'études primaire et secondaire ont un pourcentage d'utilisation non négligeable ( $26 \%$ primaire, $24 \%$ secondaire), alors que celles ayant un niveau d'études universitaire utilisent moins les plantes médicinales (9\%). Les plantes médicinales peuvent être dangereuses lorsqu'elles sont utilisées inconsciemment, et cela s'affirme chez les personnes analphabètes qui ne peuvent pas comprendre précisément les consignes verbales transmises par les herboristes et les guérisseurs. Par conséquent, elles ne respectent pas la dose, la partie utilisée et le mode de préparation des plantes médicinales, ce qui se manifeste 
et sur la sauvegarde de la biodiversité.

\section{ASPECT ETHNOBOTANIQUE ET PHARMACOLOGIQUE}

Partie utilisée : L'enquête ethnobotanique a révélé que le feuillage constitue la partie la plus utilisée dans cette région avec un pourcentage de $44,28 \%$; viennent ensuite la plante entière $(16,12 \%)$, les graines $(13,19 \%)$ et les

fleurs $(7,95 \%)$ (Fig.2). Les parties utilisées restantes à savoir tige, fruit, écorce, appareil souterrain, bulbe, et tige feuillée, sont représentées par un pourcentage cumulatif de $18,46 \%$.

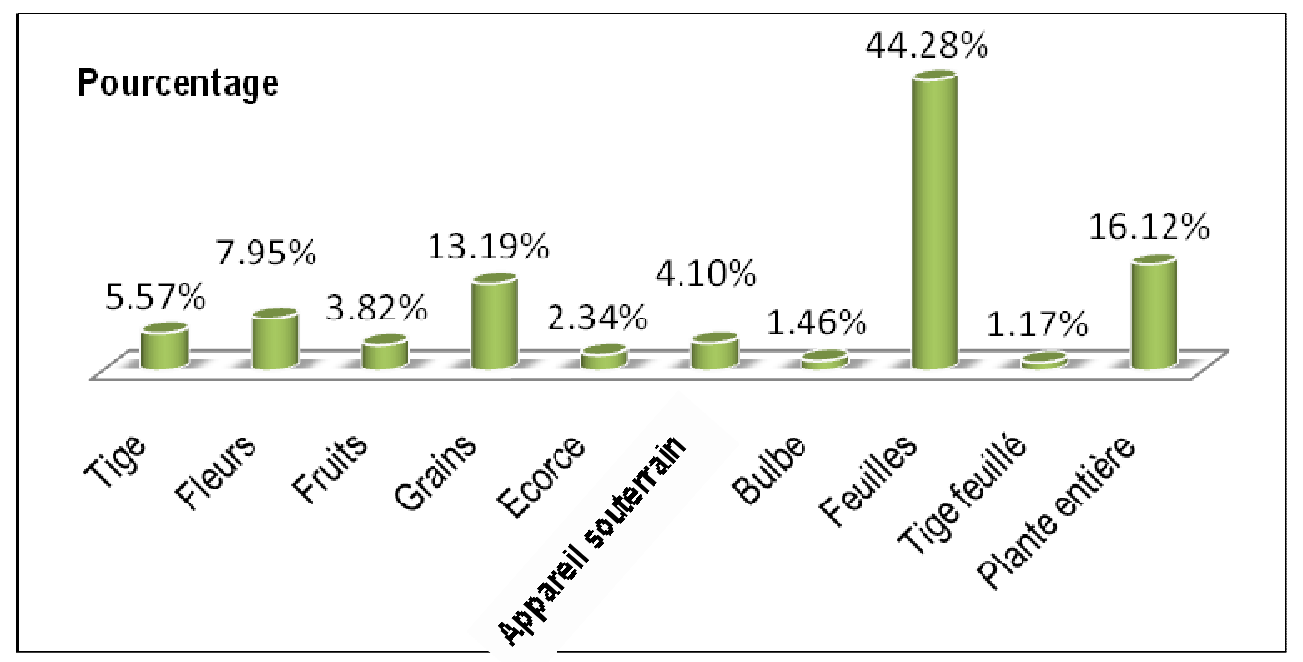

Fig.2. Répartition des différentes parties utilisées des plantes médicinales dans la Haute Moulouya.

La fréquence d'utilisation élevée de feuilles peut être expliquée par le fait qu'elles sont en même temps centrales des réactions photochimiques et réservoirs de matières organiques qui en dérivent. Elles fournissent la majorité des alcaloïdes, hétérosides et huiles essentielles (Ould El Hadj M. Didi et al., 2003). Néanmoins, la cueillette de ces organes se fait anarchiquement par les utilisateurs locaux qui ont tendance à arracher la plante entière au lieu de s'intéresser uniquement à la partie souhaitée et par conséquent cette pratique peut contribuer à la déforestation et à la dégradation des écosystèmes et des ressources naturelles.

Mode de préparation: Différentes pratiques thérapeutiques sont employées par la population locale à savoir la décoction, l'infusion, la préparation en poudre, la fumigation, le cataplasme, la macération, le cru, et la cuisson. La décoction est le mode de préparation le plus fréquent $(40,55 \%)$ dans la Haute Moulouya (Fig.3). Elle est suivie de la préparation en poudre et en cataplasme $(27,92 \%$, et $13,06 \%)$. Les autres modes (macération, infusion, cru, cuit, et fumigation) représentent $18,47 \%$. 


\section{Pourcentage}

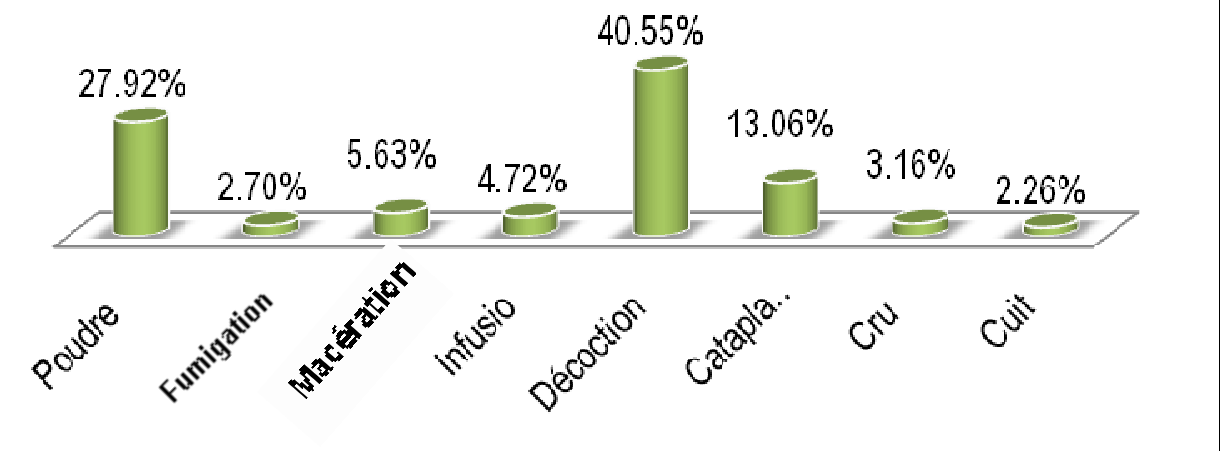

Fig.3.Répartition des différentes modes de préparation des plantes médicinales dans la Haute Moulouya.

La meilleure utilisation d'une plante serait celle qui en préserverait toutes les propriétés tous en permettant l'extraction et l'assimilation des principes actives (Dextreit, 1984). La décoction permet de réchauffer le corps et désinfecter la plante pour annuler l'effet toxique de certaines recettes, mais elle peut détruire certains principes actifs des espèces utilisées. De plus, les plantes médicinales ont des effets indésirables quand elles sont pratiquées de façon incorrecte par les patients. De ce fait, la médecine douce doit être pratiquée avec précaution et à l'intérieur des paramètres et des mesures bien précises.

Types des maladies traitées par les plantes médicinales : L'analyse ethnobotanique de l'information collectée nous a permis de répertorier un certain nombre de maladies traitées par les plantes médicinales. Les résultats représentés sur la figure 4 montrent que, la majorité des plantes médicinales interviennent principalement dans le traitement des maladies de l'appareil digestif avec un pourcentage de $45 \%$, suivies par les maladies métaboliques et les maladies ostéoarticulaires avec $13,12 \%$ chacune et les maladies dermatologique (12\%). Le reste des maladies (génitourinaires, respiratoires, neurologiques, et visuelles) représentent moins de $17 \%$, ce qui suggère que la population locale n'est pas affectée par ces derniers troubles.

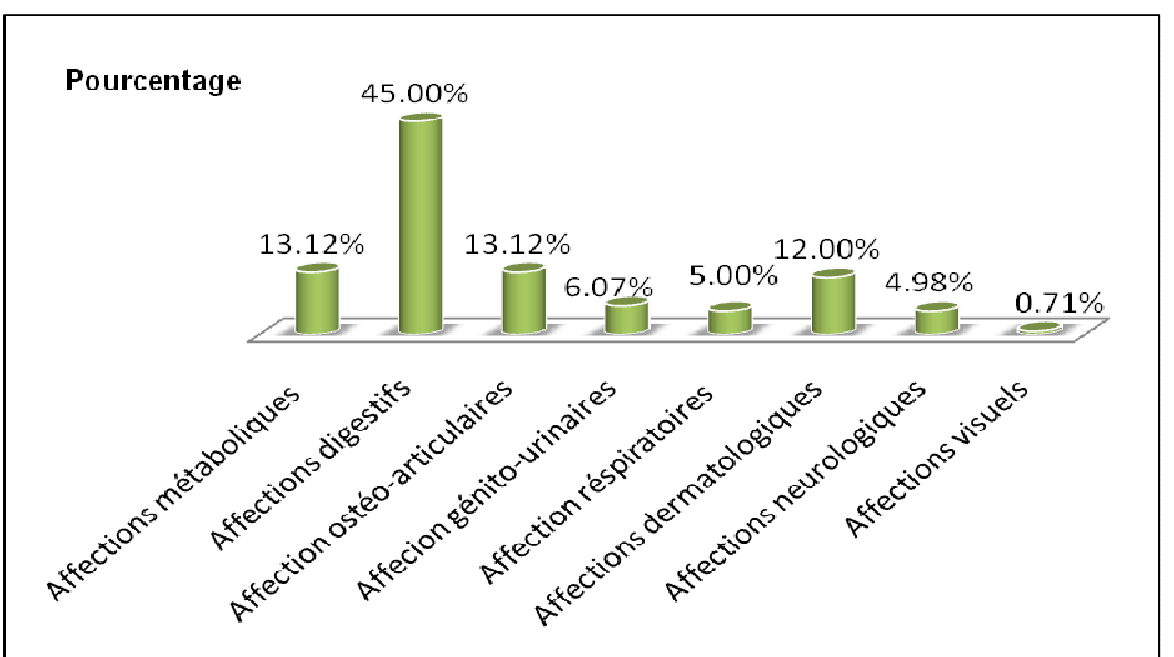

Fig.4.Répartition des différentes utilisations des plantes médicinales dans la Haute Moulouya.

La toxicité : L'analyse pharmacologique des plantes recensées, nous a permis d'identifier les plantes toxiques utilisées en pharmacopée traditionnelle dans la zone étudiée en utilisant différents ouvrages à savoir "La Pharmacopée Marocaine Traditionnelle, médecine arabe ancienne et savoir populaire» de Bellakhdar (1997) et «Les plantes médicinales du Maroc» de Sijelmassi (1993). Les résultats obtenus montrent que 40\% des plantes utilisées ont provoqué des intoxications (Fig.5). De même, la présente étude montre que la majorité des 


\section{Benlamdini et al. J. Appl. Biosci. 2014. Étude floristique et ethnobotanique de la flore médicinal du Haute}

Moulouya, Maroc

usagers de la médecine traditionnelle ignorent tout sur la toxicité de ces plantes.

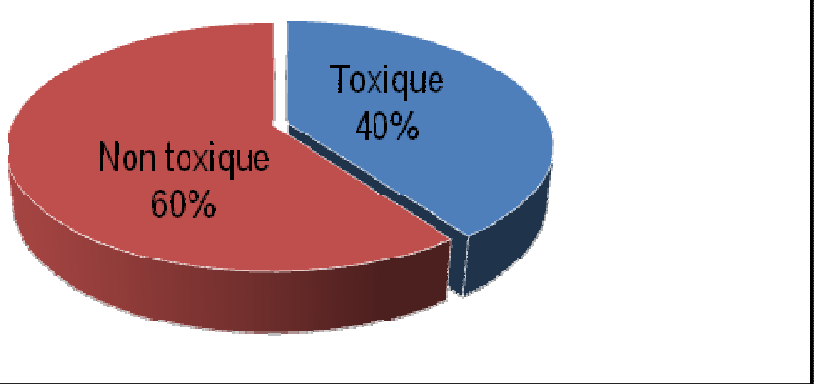

Fig.5. Répartition de pourcentage des plantes médicinales utilisées selon l'intoxication.

\section{ASPECT FLORISTIQUE}

L'analyse floristique du catalogue réalisé nous a permis de dresser la liste des espèces médicinales la plus exhaustive possible, de procéder à un classement des familles les plus représentées dans la région par le

nombre de leurs espèces et ainsi d'effectuer une comparaison significative de ces résultats à ceux obtenus par d'autres études similaires.

Tableau 2 : Nature et richesse spécifiques des familles de plantes utilisées dans la zone de la Haute Moulouya.

\begin{tabular}{|l|c|c|l|c|c|}
\hline \multicolumn{1}{|c|}{ Famille } & $\begin{array}{c}\text { Nombre } \\
\text { d'espèces }\end{array}$ & $\begin{array}{c}\text { Spécifique } \\
\%\end{array}$ & \multicolumn{1}{|c|}{ Famille } & $\begin{array}{c}\text { Nombre } \\
\text { d'espèces }\end{array}$ & $\begin{array}{c}\text { Spécifique } \\
\%\end{array}$ \\
\hline Lamiaceae & 20 & $17,39 \%$ & Capparaceae & 1 & $0,87 \%$ \\
\hline Asteraceae & 11 & $9,56 \%$ & Cannabaceae & 1 & $0,87 \%$ \\
\hline Apiaceae & 8 & $6,96 \%$ & Cucurbitaceae & 1 & $0,87 \%$ \\
\hline Fabaceae & 7 & $6,09 \%$ & Cupressaceae & 1 & $0,87 \%$ \\
\hline Rosaceae & 6 & $5,20 \%$ & Euphorbiaceae & 1 & $0,87 \%$ \\
\hline Poaceae & 5 & $4,34 \%$ & Iridaceae & 1 & $0,87 \%$ \\
\hline Myrtaceae & 3 & $2,61 \%$ & Linaceae & 1 & $0,87 \%$ \\
\hline Plantaginaceae & 3 & $2,61 \%$ & Moraceae & 1 & $0,87 \%$ \\
\hline Ranunculaceae & 3 & $2,61 \%$ & Myristicaceae & 1 & $0,87 \%$ \\
\hline Zingiberaceae & 3 & $2,61 \%$ & Nitrariaceae & 1 & $0,87 \%$ \\
\hline Amaranthaceae & 2 & $1,74 \%$ & Oleaceae & 1 & $0,87 \%$ \\
\hline Amaryllidaceae & 2 & $1,74 \%$ & Papaveraceae & 1 & $0,87 \%$ \\
\hline Apocynaceae & 2 & $1,74 \%$ & Pinaceae & 1 & $0,87 \%$ \\
\hline Brassicaceae & 2 & $1,74 \%$ & Rubiaceae & 1 & $0,87 \%$ \\
\hline Caryophyllaceae & 2 & $1,74 \%$ & Salicaceae & 1 & $0,87 \%$ \\
\hline Fagaceae & 2 & $1,74 \%$ & Schisandraceae & 1 & $0,87 \%$ \\
\hline Lauraceae & 2 & $1,74 \%$ & Solanaceae & 1 & $0,87 \%$ \\
\hline Lythraceae & 2 & $1,74 \%$ & Urticaceae & 1 & $0,87 \%$ \\
\hline Rhamnaceae & 2 & $1,74 \%$ & Verbenaceae & 1 & $0,87 \%$ \\
\hline Rutaceae & 2 & $1,74 \%$ & Vitaceae & 1 & $0,87 \%$ \\
\hline Thymelaeaceae & 1 & $1,74 \%$ & Xanthorrhoeaceae & 1 & $0,87 \%$ \\
\hline Anacardiaceae & 2 & $0,87 \%$ & Zygophyllaceae & & $0,87 \%$ \\
\hline Buxaceae & 2 & & & 1 & 1 \\
\hline
\end{tabular}

Familles botaniques les plus représentées dans la zone étudiée : Les résultats obtenus montrent que les plantes médicinales recensées dans la zone de la Haute Moulouya sont au nombre de 115 réparties en 45 familles et 99 genres (Tableau 2). De même, le nombre de plantes médicinales marocaines ne dépasse pas 600 espèces (Rejdali, 1996 ; Bammi \& Douira, 2002), soit $19,16 \%$ de la flore totale marocaine, ce qui reflète de 
façon très nette la grande richesse en plantes médicinales de cette région. Parmi les 45 familles recensées celles les plus représentées dans cette région sont: les Lamiaceae (20), les Asteraceae (11), les Apiaceae (8), les Fabaceae (7), les Rosaceae (6), et les Poaceae (5). Le résultat est conforme à celui obtenu par Kahouadji(1995) dans une autre zone du Maroc oriental. Plantes médicinales à usages très fréquent: Selon les résultats présentés dans la figure 6 , onze plantes médicinales sont relativement plus utilisées en phytothérapie traditionnelle par la population locale et la majorité de ces espèces poussent spontanément dans la région étudiée. L'espèce Origanum compactum (50) a été signalée par 50 enquêtés, suivie de Rosmarinus officinalis (40), Chenopodium ambrosioides (35), Mentha pulegium (32), Artemisia herba-alba (30), Mentha suaveolens (26), Lavandula dentatae (20), Lawsonia inermis (19), Juniperus phoenicea (15), Rosa canina (15) et Peganum harmala (14) (fig.6). Néanmoins, la récolte abusive de ces espèces peut conduire à leur disparition. Ainsi, il est nécessaire de les exploiter d'une manière raisonnable afin de les préserver et d'assurer leur durabilité.

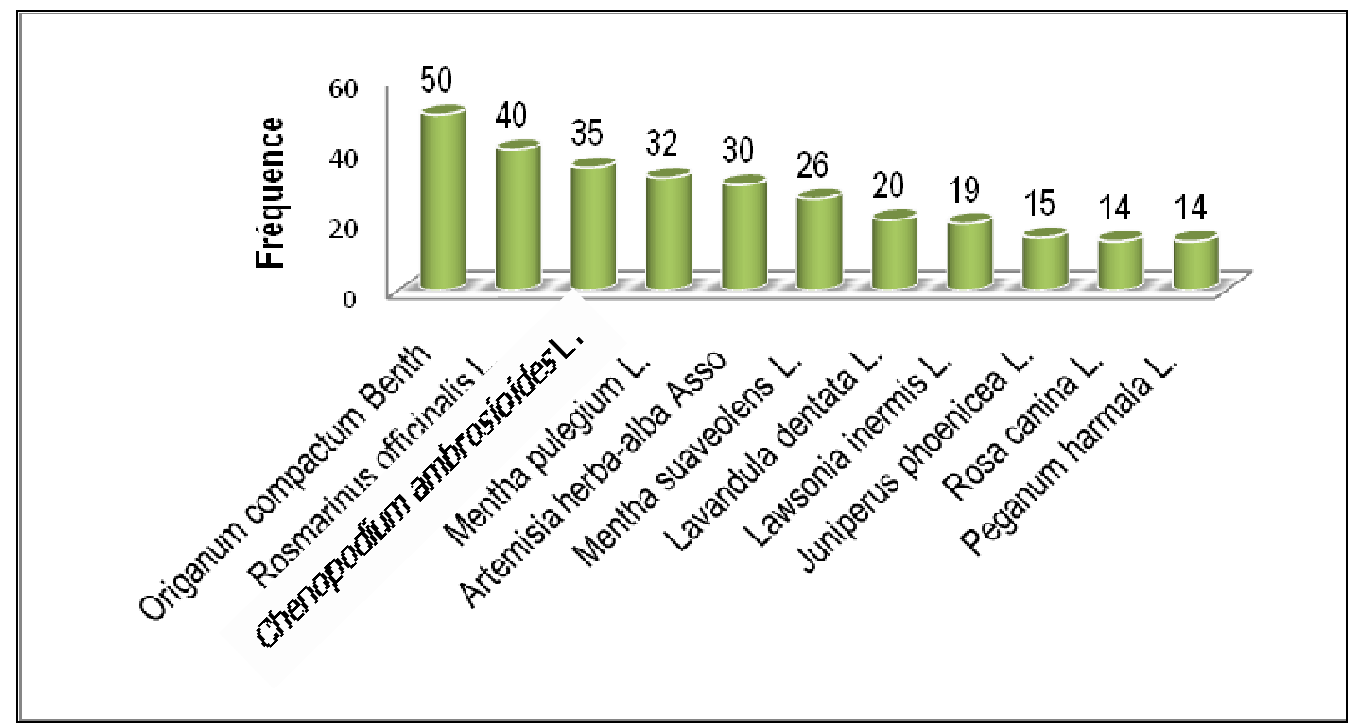

Fig. 6 : Répartition de la fréquence d'utilisation des plantes médicinales les plus utilisées.

En outre, l'air de répartition géographique de ces taxons est bien marqué par l'abondance relative des formations steppiques suivantes : Rosmarinus officinalis, Artemisia herba-alba et Peganum harmala. Ces trois espèces ont un spectre de distribution très large sur le haut bassin versant de la Moulouya, en particulier sur les massifs Ayachi et Maâsker et ses bordures. Elles s'inscrivent

\section{CONCLUSION}

Cette étude nous a permis de révéler l'importance relative accordée à la phytothérapie traditionnelle dans le système de santé à la zone de la Haute Moulouya et de confirmer que l'utilisation des plantes médicinales dans le domaine thérapeutique persiste encore malgré la révolution de la technologie médicale. De même, la collecte et l'analyse des données recueilles ont permis de transformer le savoir populaire oral dans cette région en savoir transcrit par l'établissement d'un catalogue des plantes médicinales utilisées et leurs usages dans un bioclimat semi-aride froid du montagnard méditerranéen. Cependant, la superficie des formations à armoise tend à régresser du fait de leur transformation en domaine de culture. Cette pratique a des conséquences néfastes directes sur le groupement lui-même et indirecte sur la réduction du potentiel fourrager pour les troupeaux des populations riveraines (Taleb et Fennane, 2003).

thérapeutiques. Ainsi, les résultats obtenus ont montré que les feuilles constituent les parties les plus utilisées avec un pourcentage de $44,28 \%$, et la décoction constitue l'essentiel de préparation des drogues végétales dans la phytothérapie traditionnelle. En plus, la répartition de la fréquence d'utilisation des plantes médicinales selon le groupe des maladies traitées a montré que les pathologies digestives sont les indications thérapeutiques majeures avec un pourcentage de $44 \%$. L'analyse floristique des résultats obtenus par cette étude 
a permis de recenser 115 espèces médicinales répartis en 45 familles et 99 genres avec la prédominance notamment de six familles : Lamiaceae (20), Asteraceae (11), Apiaceae (8), Fabaceae (7), Rosaceae (6), et Poaceae (5). De même, les espèces les plus utilisées dans cette région sont en nombre de 11 dont la plupart sont spontanées et relativement abondantes. Toutefois, le régime d'exploitation sévère pourra passer ces espèces dans la catégorie des espèces étrangères aux générations futures. De ce fait, la culture et la valorisation

\section{BIBLIOGRAPHIE}

Anyinam C., (1995) - Ecology and ethnomedicine. Exploring links between current environmental crisis and indigenous medical practices. Social Science and Médicine, 4, 321-329.

Aquaron M., - Relation entre les hommes et les plantes médicinales. Conférence du 18/08/2005 Les Causeries en Montagne, Sabenca de la Valéia, Barcelonnette. Site Internet: http://www.hominides.com/html/references/hom me-plantes-medicinales.php

Bammi $J$ et Douira A., (2002)-Les plantes médicinales dans la forêt de l'achach (plateau central, Maroc)-Acta BotanicaMalacitana 27: 131-145.

Bellakhdar, J., (1997) - La pharmacopée marocaine traditionnelle. Médecine arabe ancienne et savoirs populaires. Editions Le Fennec, Casablanca/ Ibis Press. 764 p. Paris.

Benkhnigue O., Zidane L., Fadli M., Elyacoubi H., Rochdi A., et Douira A., (2010-2011)- Étude ethnobotaniques des plantes médicinales dans la région de Mechraa Bel Ksiri (Région du Gharb du Maroc).Acta Bot. Barc. 53 : 191-216.

Dextreit R., (1984) - La cure végétale, Toutes les plantes pour se guérir, Vivre en harmonie, 3èmeed, 118 p.

DPAPK (Direction Provinciale de L'agriculture de la Province de Khenifra).Projet DRI-PMH de la province de khenifra, 2005. SOGREAH - Maroc développement - Rapport M3.1.73 p.

El Gasmi E., El Manssouri B., Meridekha., Tammal M., et Alwathaf Y., (2013)- Caractérisation des émergences du piémont de jbel Ayachi : contrôle structural et impact du changement climatique. Afrique science. 43-53. ISSN 1813$548 \mathrm{X}$.

El Hai H., (1968) - Biogéographie. Édit. Armand COLIN, Paris, $406 \mathrm{p}$. des plantes médicinales, ainsi que la rationalisation de la récolte des plantes spontanées s'imposent aujourd'hui afin de préserver et de réduire la pression sur les plantes médicinales les plus utilisées dans le domaine de la santé publique. Par ailleurs, la phytothérapie traditionnelle mérite encore d'autres études de prospection pour mieux recenser et identifier les plantes médicinales réparties à l'échelle nationale afin d'intégrer ce mode de soin dans le système national de santé en complément à la médecine moderne.

Emberger L. et Lemée G., (1962)- Écologie végétale. In : Les problèmes des zones arides, Édit. UNESCO, Recherche zone aride, Paris, vol. XVIII, p. 215-229.

Evans Schultes R., (1982) - Atlas Des Plantes Hallucinogènes, trad. Lepage J., 1 èr éd., FranceAmérique : Montréal, Canada, $173 \mathrm{p}$.

Fennane, M., Ibn Tattou, M., Mathez, J., Ouyahya, A. et El Oualidi, J., (1999) - Flore pratique du Maroc, Vol. I. Trav. Inst. Sci. Rabat, série botanique 36. $558 \mathrm{p}$.

Fennane, M., Ibn Tattou, J., Ouyahya, A. et El Oualidi, J., (2007) - Flore pratique du Maroc, Vol. II. Trav. Inst. Sci. Rabat, 648 p. Rabat.

Ghanmi M., Satrani B., Aberchane M., Ismaili M R., Aafi A., et El Abid A. (2011) -Plantes Aromatiques et Médicinales du Maroc, les milles et une vertu. Centre de Recherche Forestière. Rabat, Maroc, $130 \mathrm{p}$.

Guedje N.M., Ntungwen Fokunang C., Tafokou Jiofack R.B., et Fogou Dongmo R., (2010) -Opportunités d'une exploitation soutenue des plantes médicinales dans l'aménagement forestier. Int. J. Biol. Chem. Sci. 4(4) : 1346-1372, August.

Site Internet : http://indexmedicus.afro. who.int

Hamilton A., 2003- Medicinal Plants and Conservation : Issues and Approaches. Surrey (Royaume Uni) : International Plants Conservation Unit, WWFUK, 51 pages.

Hseini S et Kahouadji A., (2007)-Étude ethnobotanique de la flore médicinale dans la région de Rabat (Maroc occidental). LAZAROA 28: 79-93. 2007.

Igmoullan B., Sadki D., Fedan B., et Chellai E., (2001)Évolution géodynamique du Haut-Atlas de Midelt (Maroc) pendant le Jurassique : un exemple d'interaction entre la tectonique et l'eustatisme. Bulletin de l'Institut scientifique, 
Rabat, section Sciences de la Terre, $n^{\circ} 23,47$ 54.

Jiofack T., Fokunang C., Guedje N.M., Kemeuze V., Fongnzossie E., Nkongmeneck B.A, Mapongmetsem P.M., et Tsabang, N. (2010) Ethnobotanical uses of medicinals plants of two ethnoecological regions of Cameroon. International Journal of Medicine and Medical Sciences 2 (3) : 60-79.

Kahouadji M.S., 1995. Contribution à une étude ethnobotanique des plantes médicinales dans le Maroc oriental, Thèse de Doctorat 3ème cycle, Université Mohamed I, Fac. Sci. Oujda, 220 p.

Kahouadji A., (1986) - Recherches floristiques sur le massif montagneux des Beni Snassene (Maroc oriental), Université des Sciences et Techniques du Languedoc, Montpellier, $235 \mathrm{pp}$.

Lahsissene H., Kahouadji A., Tijane M., et Hseini S., (2009)-Catalogue des plantes médicinales utilisées dans la région de zaër (Maroc occidental).Lejeunia, BE ISSN 0457-4184.

Mpondo M.E., DIBONG D.S., PRISO R.J., NGOYE A., et LADOH YEMEDA C.F., (2012) -État actuel de la médecine traditionnelle dans le système de santé des populations rurales et urbaines de Douala (Cameroun). Journal of Applied Biosciences 55 : 4036- 4045.

Monographie de la région Meknès-Tafilalet., (2009)Ministère de l'intérieur.
Ould El Hadj M. Didi, Hadj-Mahammed M., Zabeirou H., (2003) - Place des plantes spontanées dans la médicine traditionnelle de la région d'Ouargla (Sahara septentrional est). Courrier du Savoir $\mathrm{N}^{\circ} 03$, pp. 47-51.

Peyron M., (1976)- Habitat rural et vie montagnarde dans le Haut Atlas de Midelt (Maroc). Revue de géographie alpine. Tome $64 \mathrm{~N}^{\circ} 3$. pp. 327-363.

Rejdali, M., 1996- La flore du Maroc: état actuel et perspectives de conservation. Diversité biologique et valorisation des plantes médicinales. Actes. éd. 17-22.

Rhanem M., (2009)- L'alfa (Stipa tenacissima L.) dans la plaine de Midelt (haut bassin versant de la Moulouya) - Éléments de climatologie. PhysioGéo - Géographie Physique et Environnement, vol. III, p. 1-20.

Sijelmassi A., 1993 - Les plantes médicinales du Maroc, 6ème édition. Fennec. Casablanca. $285 \mathrm{p}$.

Scherrer A.M., Motti R., et Weckerle C. S., (2005) Traditional plant use in the areas of Monte Vesole and Ascea, Cilento National Park (Campania, Southern Italy) - J. Ethnopharmacol. 97: 129-143.

Sofowora A., (1993) - Medicinal plants and traditional medicine in Africa, 2 - Spectrum Books Limited, Ibadan, Nigeria, 289p.

Taleb M et Fennane M., (2003)- Étude des groupements steppiques du Parc national du Haut Atlas oriental et ses bordures (Maroc).

\section{ANNEXE}

Catalogue des plantes médicinales utilisées dans la zone de Haute Moulouya

\begin{tabular}{|c|c|c|c|c|}
\hline Famille & $\begin{array}{c}\text { Espèce végétale } \\
\text { Nom scientifique et } \\
\text { vernaculaire (français, arabe } \\
\text { et amazigh) }\end{array}$ & $\begin{array}{l}\text { Partie } \\
\text { utilisée }\end{array}$ & $\begin{array}{l}\text { Mode de } \\
\text { préparation }\end{array}$ & $\begin{array}{l}\text { Administration et Usages } \\
\text { thérapeutique locaux }\end{array}$ \\
\hline \multirow[t]{2}{*}{ Amaranthaceae } & $\begin{array}{l}\text { Chenopodium ambrosioides L. } \\
\left({ }^{*}\right) \\
\text { Ansérine / Mkhinza }\end{array}$ & $\begin{array}{l}\mathrm{Fe} \\
\mathrm{Fe}\end{array}$ & $\begin{array}{l}\text { Déc + Jus } \\
\text { d'orange } \\
\text { Cat }\end{array}$ & $\begin{array}{l}\text { Or: Stomachiques. Fébrifuge. } \\
\text { ApT : Fébrifuge. }\end{array}$ \\
\hline & $\begin{array}{l}\text { Haloxylon scoparium Pomel }\left({ }^{*}\right) \\
\text { Saligne à balai / Rremt }\end{array}$ & HE & $\mathrm{Cr}$ & UE : contre le rhumatisme. \\
\hline \multirow[t]{2}{*}{ Amaryllidaceae } & $\begin{array}{l}\text { Allium cepa L. } \\
\text { Oignon/ I-beslâ / âzlim }\end{array}$ & $\mathrm{Bb}$ & Cr en Mac & Or: Antipyrétique. \\
\hline & $\begin{array}{l}\text { Allium sativum L. } \\
\text { Ail cultivé /Toûma / Tiskert }\end{array}$ & $\begin{array}{l}\mathrm{Bb} \\
\mathrm{Bb} \\
\mathrm{Bb}\end{array}$ & $\begin{array}{c}\text { CrDH ou CtDH } \\
\text { CrDH } \\
\text { CrDH en Cat }\end{array}$ & $\begin{array}{l}\text { Or: Antipyrétique, vermifuge. } \\
\text { Col: Contre les affections } \\
\text { visuelles. } \\
\text { ApCCC: Assouplissant, et } \\
\text { antipelliculaire. }\end{array}$ \\
\hline Anacardiaceae & $\begin{array}{l}\text { Pistacia lentiscus L. } \\
\text { Lentisque /Drou }\end{array}$ & $\mathrm{Fe}$ & Déc & Or: Stomachique. \\
\hline
\end{tabular}


Benlamdini et al. J. Appl. Biosci. 2014. Étude floristique et ethnobotanique de la flore médicinal du Haute Moulouya, Maroc

\begin{tabular}{|c|c|c|c|c|}
\hline \multirow[t]{8}{*}{ Apiaceae } & $\begin{array}{l}\text { Ammodaucus leucotrichus } \\
\text { Coss. \&Dur. } \\
\text { Cumin velu /Kammûn es-sofi }\end{array}$ & $\mathrm{Fr}$ & Déc & Or : Stomachique. \\
\hline & $\begin{array}{l}\text { Carum carvi L. }\left({ }^{*}\right) \\
\text { Carvi Karwiya }\end{array}$ & $\mathrm{Gr}$ & $\mathrm{Cr}$ & Or: Carminatif. \\
\hline & $\begin{array}{l}\text { Coriandrum sativum L. } \\
\text { Coriandre cultivé /Kozber }\end{array}$ & $\begin{array}{l}\mathrm{Gr} \\
\mathrm{Gr}\end{array}$ & $\begin{array}{l}\text { Fum } \\
\text { Déc }\end{array}$ & $\begin{array}{l}\text { Inh : Contre le } \\
\text { refroidissement. } \\
\text { Or : Stomachique. Contre les } \\
\text { calculs rénaux et la diarrhée. }\end{array}$ \\
\hline & $\begin{array}{l}\text { Cuminum cyminum L. } \\
\text { Cumin /Kammûn }\end{array}$ & $\mathrm{Gr}$ & $\mathrm{Ct}$ & $\begin{array}{lll}\text { Orl Me : } & \text { Contre } & \text { la } \\
\text { constipation. } & & \end{array}$ \\
\hline & $\begin{array}{l}\text { Daucus Carota L. } \\
\text { Carotte /Hizzû }\end{array}$ & $\mathrm{Fr}$ & Jus & $\begin{array}{l}\text { Or: Anti- diarrhéique, } \\
\text { ténifuge. }\end{array}$ \\
\hline & $\begin{array}{l}\text { Foeniculum vulgare Mill. }{ }^{*} \text { ) } \\
\text { Fenouil sauvage /Besbass } \\
\text { berri / Nâfaa }\end{array}$ & $\mathrm{Gr}$ & Déc & $\begin{array}{l}\text { Or: Stomachique. Contre le } \\
\text { refroidissement. }\end{array}$ \\
\hline & $\begin{array}{l}\text { Petroselinum sativum Hoffm. } \\
\text { Persil /Maàdnoûs }\end{array}$ & PA & Déc & $\begin{array}{l}\text { Or: Contre les lithiases } \\
\text { rénales. }\end{array}$ \\
\hline & $\begin{array}{l}\text { Pimpinella anisum L. }\left({ }^{*}\right) \\
\text { Anis /Yansoune /Habet hlâwa }\end{array}$ & $\begin{array}{l}\mathrm{Gr} \\
\mathrm{Gr}\end{array}$ & $\begin{array}{l}\text { Inf } \\
\text { Pdr }\end{array}$ & $\begin{array}{l}\text { Or: Stomachique. } \\
\text { Or: Contre l'insomnie }\end{array}$ \\
\hline \multirow[t]{2}{*}{ Apocynaceae } & $\begin{array}{l}\text { Caralluma europaea (Guss.) } \\
\text { N.E.Br.ssp.maroccana } \\
\text { (Hooker f.) Maire. } \\
\text { Caralluma /Daghmoûss }\end{array}$ & $\begin{array}{l}\text { Gr } \\
\text { Gr }\end{array}$ & $\begin{array}{l}\text { Pdr } \\
\text { Inf }\end{array}$ & $\begin{array}{l}\text { Or: Contre l'insomnie. } \\
\text { Or: Stomachique. }\end{array}$ \\
\hline & $\begin{array}{l}\text { Nerium oleander L. }\left({ }^{*}\right) \\
\text { Laurier -rose /Defla /Alili }\end{array}$ & $\mathrm{Fe}$ & Inf & BaPds : Antidiabétique. \\
\hline \multirow[t]{10}{*}{ Asteraceae } & $\begin{array}{l}\text { Anacyclus pyrethrum DC. }\left(^{*}\right) \\
\text { Pyrèthre d'Afrique IAerq } \\
\text { Chleuh/ Tiguentest }\end{array}$ & $T$ & PdrDM & $\begin{array}{l}\text { Or: Contre les kystes de } \\
\text { l'appareil génital. }\end{array}$ \\
\hline & $\begin{array}{l}\text { Artemisia absinthium L. }\left(^{*}\right) \\
\text { Absinthe /Chiba }\end{array}$ & TFe & Déc & Or : contre le refroidissement. \\
\hline & $\begin{array}{l}\text { Artemisia herba-alba Asso }\left(^{*}\right) \\
\text { Armoise blanche /Chih / Izri }\end{array}$ & PA & Déc & $\begin{array}{l}\text { Or: Antitussif, antidiabétique, } \\
\text { contre le refroidissement. }\end{array}$ \\
\hline & $\begin{array}{l}\text { Artemisia Mesatlantica Maire. } \\
\left({ }^{*}\right) \\
\text { Armoise bleu /Chih/ Ifsi }\end{array}$ & PA & Déc & $\begin{array}{l}\text { Or: Antitussif, antidiabétique, } \\
\text { contre le refroidissement. }\end{array}$ \\
\hline & $\begin{array}{l}\text { Atractylis gummifera L. }\left(^{*}\right) \\
\text { Atractyle /Dâd/ Dlagh }\end{array}$ & $\mathbf{R a}$ & Pdr & Or : Facilite l'accouchement. \\
\hline & $\begin{array}{l}\text { Chrysanthemum coronarium L. } \\
\text { Marguerite en couronne / El } \\
\text { Okhouane/ l'gentus }\end{array}$ & PA & Pdr & Or : Somnifère. \\
\hline & $\begin{array}{l}\text { Dittrichia viscosa (L.) Greuter } \\
\text { Inule visqueuse /Terrahla/ } \\
\text { Magraman }\end{array}$ & $\begin{array}{l}\mathrm{Fe}+\mathrm{Fe} \\
\text { de } \\
\text { l'origan }\end{array}$ & Déc & $\begin{array}{l}\text { Or: Anti-diarrhéique, et } \\
\text { contre la fièvre typhoïde. }\end{array}$ \\
\hline & $\begin{array}{l}\left.\text { Lactuca serriola L. }{ }^{*}\right) \\
\text { Laituescariol /Hedba Ibldia / } \\
\text { Tign-sem }\end{array}$ & $\mathrm{Fe}$ & Cat & $\begin{array}{l}\text { ApL: Antipoison de des } \\
\text { morsures de serpents et des } \\
\text { piqures des scorpions. }\end{array}$ \\
\hline & $\begin{array}{l}\text { Matricaria chamomilla L. } \\
\text { Camomille sauvage /Babounje }\end{array}$ & $\mathrm{Fe}$ & $\operatorname{lnf}$ & Or: Stomachique. \\
\hline & Ormenis scariosa (Ball) Litard. & PA & Déc & Stomachique, \\
\hline
\end{tabular}


Benlamdini et al. J. Appl. Biosci. 2014. Étude floristique et ethnobotanique de la flore médicinal du Haute Moulouya, Maroc

\begin{tabular}{|c|c|c|c|c|}
\hline & $\begin{array}{l}\text { \& Maire } \\
\text { Ormenis /Ghartoufa / Irezgui }\end{array}$ & & & emménagogue et vermifuge. \\
\hline & $\begin{array}{l}\text { Santolina rosmarinifolia L. } \\
\text { Santoline à feuilles de romarin } \\
\text { /Ouezouaza }\end{array}$ & $\mathrm{Fe}$ & Déc & Or : Carminatif. \\
\hline \multirow[t]{2}{*}{ Brassicaceae } & $\begin{array}{l}\text { Brassicarapa L. }\left(^{*}\right) \\
\text { Navet potager/Leffet }\end{array}$ & $\mathrm{Fe}$ & Déc & Or : Antidiabétique. \\
\hline & $\begin{array}{l}\text { Lepidium sativum L. }\left(^{*}\right) \\
\text { Cresson alénois } / \text { Heb-rchad }\end{array}$ & $\mathrm{Gr}$ & Mac ou Ct & Or: Stomachique. \\
\hline Buxaceae & $\begin{array}{l}\text { Buxus balearica Lam. } \\
\text { Buis des baléares /Azazer }\end{array}$ & $\mathrm{Fe}$ & Déc & Or : Anti-refroidissement. \\
\hline Capparaceae & $\begin{array}{l}\text { Capparis spinosa L. } \\
\text { Cậprier /Kabbar / Teïloulout }\end{array}$ & $\mathrm{Fe}$ & Déc & $\begin{array}{l}\text { Or: Contre l'asthme et } \\
\text { l'allergie. }\end{array}$ \\
\hline Cannabaceae & $\begin{array}{l}\text { Cannabis sativa L. }\left(^{*}\right) \\
\text { Chanvre cultivé/Kife }\end{array}$ & $\mathrm{Gr}+\mathrm{T}$ & PdrDH en Cat & ApC : Assouplissant. \\
\hline \multirow[t]{2}{*}{ Caryophyllaceae } & $\begin{array}{l}\text { Herniaria glabra } \mathrm{L} \text {. } \\
\text { Herniaire glabre /Herras lehjar }\end{array}$ & $\begin{array}{l}\text { PA+ } \\
\text { Ra de } \\
\text { persil }\end{array}$ & Déc & $\begin{array}{l}\text { Or: Contre les lithiases } \\
\text { rénales. }\end{array}$ \\
\hline & \begin{tabular}{ll}
\multicolumn{2}{l}{ Saponnaria Vaccaria L. $\left(^{*}\right)$} \\
saponaire des & vaches \\
/Tighighechte &
\end{tabular} & $\mathrm{Fe}$ & Cat & $\begin{array}{l}\text { ApL: Contre la gale et les } \\
\text { affections contagieuses de la } \\
\text { peau. }\end{array}$ \\
\hline Cucurbitaceae & $\begin{array}{l}\text { Citrullus colocynthis (L.) } \\
\text { Schrad. } \\
\text { Coloquinte /Lhdej / Taferzizte }\end{array}$ & $\mathrm{Fr}$ & $\mathrm{Cr}+\mathrm{Fum}$ & Or/Inh : Facilite la délivrance. \\
\hline Cupressaceae & $\begin{array}{l}\text { Juniperus phoenicea L. } \\
\text { Genévrier de Phénicie /Ar'âr/ } \\
\text { Taqga/ Tahlaoute }\end{array}$ & TFe & PdrDM & $\begin{array}{l}\text { Or: Stomachique, contre la } \\
\text { constipation. }\end{array}$ \\
\hline Euphorbiaceae & $\begin{array}{l}\text { Mercurialis annua L. (*) } \\
\text { Mercuriale annuelle /Hurryqal- } \\
\text { melsâ }\end{array}$ & $\mathrm{Fe}$ & Inf & Or: Stomachique. \\
\hline \multirow[t]{7}{*}{ Fabaceae } & $\begin{array}{l}\text { Adenocarpus bacquei Batt. } \\
\text { \&Pitard(*) } \\
\text { Adenocarpus /Tagoultemte }\end{array}$ & $\mathrm{Fe}$ & Déc & Or: Contre le rhumatisme. \\
\hline & $\begin{array}{l}\text { Cassia senna L. } \\
\text { Cassia / Sna'a }\end{array}$ & $\mathrm{Fe}$ & Déc & Or: Stomachique. \\
\hline & $\begin{array}{l}\text { Ceratonia siliqua L. } \\
\text { Caroubier /El kharroub/Tikidit }\end{array}$ & $\mathrm{Fe}$ & PdrDM & Or: Stomachique. \\
\hline & $\begin{array}{l}\text { Medicago sativa L. } \\
\text { Luzerne cultivée /Fsa al- } \\
\text { berriya }\end{array}$ & $\mathrm{Fe}$ & Pdr & Or: Stomachique. \\
\hline & $\begin{array}{l}\text { Retama sphaerocarpa } \\
\text { (L.) Boiss.(*) } \\
\text { Retama amarilla /Rrtem / llbi }\end{array}$ & HE & $\mathrm{Cr}$ & UE : Contre le rhumatisme. \\
\hline & $\begin{array}{l}\text { Trigonellafoenum graecum L. } \\
\text { Fenugrec /Helba / Tifidass }\end{array}$ & $\mathrm{Bb}$ & MélDH en Cat & $\begin{array}{l}\text { ApL: Contre les maladies } \\
\text { des yeux. } \\
\text { ApCCC : antichute. }\end{array}$ \\
\hline & $\begin{array}{l}\text { Vicia fabaL. }\left({ }^{*}\right) \\
\text { Fève /Fûl/ îbaûn }\end{array}$ & $\mathrm{Ra}$ & Pdr en Mac & Or: Contre l'asthme. \\
\hline Fagaceae & $\begin{array}{l}\text { Quercus rotundifolia Lamk. } \\
\text { Chênevert /Al-ballût/ Kerrouch }\end{array}$ & $\mathbf{R a}$ & Déc & Or: Stomachique. \\
\hline
\end{tabular}


Benlamdini et al. J. Appl. Biosci. 2014. Étude floristique et ethnobotanique de la flore médicinal du Haute Moulouya, Maroc

\begin{tabular}{|c|c|c|c|c|}
\hline & $\begin{array}{l}\text { Quercus suber L. }\left(^{*}\right) \\
\text { Chêne liège /Chajar El Ferchi/ } \\
\text { Fernâne }\end{array}$ & GId & Pdr & $\begin{array}{l}\text { Or: Contre l'impuissance } \\
\text { sexuelle. }\end{array}$ \\
\hline Iridaceae & $\begin{array}{l}\text { Crocus sativus } \mathrm{L} \text {. } \\
\text { Safran cultivé/Za'afran Ihôr }\end{array}$ & Stg & $\mathrm{Ct}$ & Or : Facilite l'accouchement. \\
\hline \multirow[t]{12}{*}{ Lamiaceae } & $\begin{array}{l}\text { Ajugaiva (L.) Schreb. } \\
\text { Ivette musquée /Chendgoura/ } \\
\text { Tûf Telba }\end{array}$ & $\mathrm{Fe}$ & Déc & $\begin{array}{l}\text { Or: Contre les affections } \\
\text { rénales. }\end{array}$ \\
\hline & $\begin{array}{l}\left.\text { Lavandula dentata } \mathrm{L} .{ }^{*}\right) \\
\text { Lavande vraie Khzama I } \\
\text { Temezriya }\end{array}$ & $\begin{array}{l}\mathrm{Fe} \\
\mathrm{Fe}\end{array}$ & $\begin{array}{c}\text { Déc } \\
\text { PdrDHen en } \\
\text { Cat }\end{array}$ & $\begin{array}{l}\text { Or: Stomachique, } \\
\text { emménagogue, contre le } \\
\text { refroidissement. } \\
\text { ApCCC: Adoucissante, } \\
\text { antichute. }\end{array}$ \\
\hline & $\begin{array}{l}\text { Lavandula tenuisecta Coss. ex } \\
\text { Ball } \\
\text { Lavande à feuilles dentées } \\
\text { /Kohhayla /Tiguizte }\end{array}$ & SFI & Déc & Or: Ténifuge. \\
\hline & $\begin{array}{l}\text { Marrubium vulgare L. }\left(^{*}\right) \\
\text { Marrube blanc /Merrîwut /lfzi }\end{array}$ & $\mathrm{Fe}$ & Pdr & Or: Ténifuge. \\
\hline & $\begin{array}{l}\text { Marrubium multibracteatum } \\
\text { Humbert \& Maire }\left(^{*}\right) \\
\text { Marrube /Merrîwut / Ifzi }\end{array}$ & $\mathrm{Fe}$ & Pdr & Or : Ténifuge, stomachique. \\
\hline & $\begin{array}{l}\text { Mentha pulegium L. (*) } \\
\text { Menthe pouliot /Fliyyo dial mâ } \\
\text { /Tazoukennit }\end{array}$ & $\mathrm{Fe}$ & $\begin{array}{l}\text { Déc } \\
\text { Pdr }\end{array}$ & $\begin{array}{l}\text { Or: Stomachique. Fébrifuge. } \\
\text { Contre le refroidissement, et } \\
\text { contre les affections } \\
\text { respiratoires (grippe, rhume, } \\
\text { et bronchite). } \\
\text { ApL : Détersif. }\end{array}$ \\
\hline & $\begin{array}{l}\text { Mentha suaveolens Ehrh. } \\
\text { Menthe à feuilles rondes } \\
\text { /Marseta /Timersad }\end{array}$ & $\mathrm{Fe}$ & Déc & $\begin{array}{l}\text { Or: Stomachique. Contre le } \\
\text { refroidissement. } \\
\text { Emménagogue. }\end{array}$ \\
\hline & $\begin{array}{l}\text { Mentha spicata L. } \\
\text { Menthe en épi /Na'na' }\end{array}$ & $\mathrm{Fe}$ & Déc & Or : Stomachique. \\
\hline & $\begin{array}{l}\text { Mentha } x \text { piperita } \mathrm{L} \text {. } \\
\text { Menthe poivrée Na'na' } \\
\text { lbasatine }\end{array}$ & $\mathrm{Fe}$ & Déc & Or : Stomachique. \\
\hline & $\begin{array}{l}\text { Origanum compactum Benth. } \\
\left({ }^{*}\right) \\
\text { Origan à inflorescence } \\
\text { compacte /Za'tar/ Azoukni }\end{array}$ & $\mathrm{Fe}$ & PdrDH en Cat & $\begin{array}{l}\text { Or: Stomachique. Fébrifuge. } \\
\text { Contre le refroidissement, et } \\
\text { contre les affections } \\
\text { respiratoires (grippe, rhume, } \\
\text { et bronchite). } \\
\text { ApC : Assouplissant. } \\
\text { antipelliculaire. }\end{array}$ \\
\hline & $\begin{array}{l}\text { Origanum majorana L. } \\
\text { Marjolaine /Mardadouch }\end{array}$ & $\mathrm{Fe}$ & Déc & Or : Anti-refroidissement. \\
\hline & $\begin{array}{l}\text { Rosmarinus officinalis L. }\left({ }^{*}\right) \\
\text { Romarin /Azir/Assir }\end{array}$ & $\begin{array}{l}\text { TFe } \\
\text { TFe } \\
\text { HE }\end{array}$ & $\begin{array}{c}\text { Déc ou Inf } \\
\text { Cat } \\
\text { Cr }\end{array}$ & $\begin{array}{l}\text { Or: Stomachique. Fébrifuge. } \\
\text { Contre le refroidissement. } \\
\text { Facilite l'accouchement. } \\
\text { ApL: Contre l'eczéma des } \\
\text { pieds, et le refroidissement. }\end{array}$ \\
\hline
\end{tabular}


Benlamdini et al. J. Appl. Biosci. 2014. Étude floristique et ethnobotanique de la flore médicinal du Haute Moulouya, Maroc

\begin{tabular}{|c|c|c|c|c|}
\hline & & HE & $\mathrm{Cr}$ & $\begin{array}{l}\text { UE : Détersif. Antiseptiques. } \\
\text { Or : Béchique. }\end{array}$ \\
\hline & $\begin{array}{l}\text { Salvia officinalis L. }\left(^{*}\right) \\
\text { Sauge-officinale /Sâlmiya }\end{array}$ & $\mathrm{Fe}$ & Déc & Or : Hypoglycémiant. \\
\hline & $\begin{array}{l}\text { Salvia verbenaca (L.) Briq. } \\
\text { Sauge- verveine /Hiyyata }\end{array}$ & $\mathrm{Fe}$ & Cat & $\begin{array}{l}\text { Or: Contre les blessures et } \\
\text { les abcès. Astringente. }\end{array}$ \\
\hline & $\begin{array}{l}\text { Sideritis incana L. } \\
\text { Sideritis /Agoûltme }\end{array}$ & PA & Déc & Or: Contre les rhumatismes. \\
\hline & $\begin{array}{l}\text { Teucrium fruticans } \mathrm{L} . \\
\text { Germandrée arbustive /Miro }\end{array}$ & $\mathrm{Fe}$ & Déc & $\begin{array}{l}\text { Or: Stomachique. Contre le } \\
\text { refroidissement. }\end{array}$ \\
\hline & $\begin{array}{l}\text { Teucrium polium L. } \\
\text { Germandrée blanc-grisâtre } \\
\text { /Ja'idiya / Tayrart }\end{array}$ & $\mathrm{Fe}$ & Déc & $\begin{array}{l}\text { BaBo : Pour calmer l'irritation } \\
\text { et la douleur des gencives. }\end{array}$ \\
\hline & $\begin{array}{l}\text { Thymus zygis L. } \\
\text { Thym /Za'aytra / Azoukenni }\end{array}$ & Fe & Déc & $\begin{array}{l}\text { Or: Contre } \quad \text { le } \\
\text { refroidissement. Carminatif. } \\
\text { Anti-diarrhéique. }\end{array}$ \\
\hline & $\begin{array}{l}\text { Thymus pallidus Butt. } \\
\text { Thym /Za'aytra / Azoukenni }\end{array}$ & $\mathrm{Fe}$ & Déc & $\begin{array}{lll}\text { Or: } & \text { Contre } & \text { le } \\
\text { refroidissement. } & \\
\end{array}$ \\
\hline & $\begin{array}{l}\text { Ziziphora hispanica L. } \\
\text { Ziziphora /Fliyo diâl-berr I } \\
\text { Taflyoute }\end{array}$ & $\mathrm{Fe}$ & Déc & $\begin{array}{lll}\text { Or: } & \text { Contre } & \text { le } \\
\text { refroidissement. stomachique. }\end{array}$ \\
\hline Lauraceae & $\begin{array}{l}\text { Cinnamomum verum J.PresI } \\
\text { Cannelier de Ceylan /Qarfa }\end{array}$ & Ec & Pdr & $\begin{array}{l}\text { Or: Calmer les douleurs de } \\
\text { l'accouchement. }\end{array}$ \\
\hline & $\begin{array}{l}\text { Laurus nobilis } \mathrm{L} \text {. } \\
\text { Laurier noble /warkat sidna } \\
\text { moussa }\end{array}$ & $\mathrm{Fe}$ & Déc & $\begin{array}{l}\text { Or: Contre les maladies } \\
\text { génito-urinaires. }\end{array}$ \\
\hline Linaceae & $\begin{array}{l}\text { Linum usitatissimum L. }\left(^{*}\right) \\
\text { Lin /Zerî’at I-kettân }\end{array}$ & $\mathrm{Gr}$ & Pdr & Or: Stomachique. \\
\hline Lythraceae & $\begin{array}{l}\text { Lawsonia inermis L. } \\
\text { Henné /Henna }\end{array}$ & $\begin{array}{l}\mathrm{Fe} \\
\mathrm{Fe}\end{array}$ & $\begin{array}{l}\text { Pdr } \\
\text { Pdr }\end{array}$ & $\begin{array}{l}\text { ApL: Détersif, et contre } \\
\text { l'eczéma. } \\
\text { ApCCC : Assouplissant, et } \\
\text { antipelliculaire. }\end{array}$ \\
\hline & $\begin{array}{l}\text { Punica granatum L. } \\
\text { Grenadier /Er-rummân }\end{array}$ & EcDFr & Déc & Or: Stomachique. \\
\hline Moraceae & $\begin{array}{l}\text { Ficus carica L. } \\
\text { Figuier /Karmôs / Sheriha }\end{array}$ & $\mathrm{Fr}$ & PdrDH & $\begin{array}{l}\text { Or: Contre l'impuissance } \\
\text { sexuelle et le refroidissement. }\end{array}$ \\
\hline Myristicaceae & $\begin{array}{l}\text { Myristic afragrans Houtt. }{ }^{*} \text { ) } \\
\text { Muscadier /Bsibissa / Lggouza }\end{array}$ & TgmDNx & $\mathrm{Ct}$ & Or : Somnifères et antitussif. \\
\hline Myrtaceae & $\begin{array}{l}\text { Eucalyptus globulus Labill. } \\
\text { Eucalyptus /Kelitto/ kalitus }\end{array}$ & TFe & Fum & Inh : Contre la grippe. \\
\hline & $\begin{array}{l}\text { Eugenia caryophyllata Thunb } \\
\text { Giroflier /Qoronfel }\end{array}$ & Cls & $\begin{array}{l}\text { PdrDHen en } \\
\text { Cat }\end{array}$ & $\begin{array}{l}\text { ApCCC: Adoucissante. } \\
\text { Antichute. Analgésique. }\end{array}$ \\
\hline & $\begin{array}{l}\text { Myrtus communis L. }\left(^{*}\right) \\
\text { Myrte /Rihân }\end{array}$ & $\mathrm{Fe}$ & $\begin{array}{l}\text { PdrDHen en } \\
\text { Cat }\end{array}$ & $\begin{array}{l}\text { ApCCC : Adoucissante. Anti- } \\
\text { chute. Démêleur. }\end{array}$ \\
\hline Nitrariaceae & $\begin{array}{l}\text { Peganum harmala L. }\left(^{*}\right) \\
\text { Harmel /Harmel }\end{array}$ & $\begin{array}{l}\mathrm{Gr} \\
\mathrm{Gr} \\
\mathrm{Gr}\end{array}$ & $\begin{array}{l}\text { Fum } \\
\text { PdrDH en Cat } \\
\text { PdrDL }\end{array}$ & $\begin{array}{l}\text { Inh: le Contre } \\
\text { refroidissement. } \\
\text { ApC : Assouplissant. } \\
\text { Or: Fébrifuge. Stomachique. } \\
\text { Contre les maux de tête. }\end{array}$ \\
\hline Oleac & Olea europeae L. & $\mathrm{Fe}$ & Inf & Hypotenseur. \\
\hline
\end{tabular}


Benlamdini et al. J. Appl. Biosci. 2014. Étude floristique et ethnobotanique de la flore médicinal du Haute Moulouya, Maroc

\begin{tabular}{|c|c|c|c|c|}
\hline & Olivier /Zitoun & & & Antidiabétique. \\
\hline Papaveraceae & $\begin{array}{l}\text { Papaver rhoeas L. }\left(^{*}\right) \\
\text { Coquelicot /Belaâmane }\end{array}$ & $\mathrm{Fe}$ & Déc & Or : Stomachique. sédatif. \\
\hline Pinaceae & $\begin{array}{l}\text { Pinus halepensis Mill. (*) } \\
\text { Pin d'Alep /Taydâ }\end{array}$ & $\mathrm{Fe}$ & Déc & $\begin{array}{l}\text { Or: Contre le maux des } \\
\text { dents. }\end{array}$ \\
\hline \multirow[t]{3}{*}{ Plantaginaceae } & $\begin{array}{l}\text { Kickxia commutata (Bernh. ex } \\
\text { Rchb.) Fritsch } \\
\text { Linaire grecque/Astfal }\end{array}$ & $\mathrm{Fe}$ & Cat & ApL : Détersif. \\
\hline & $\begin{array}{l}\text { Plantago coronopus L. }\left({ }^{*}\right) \\
\text { Plantain corne de cerf /Rjil-el- } \\
\text { gorâb }\end{array}$ & $\mathrm{Fe}$ & Cat & $\begin{array}{l}\text { ApL: } \quad \text { Anti-inflammatoire. } \\
\text { Antiprurigineuse. }\end{array}$ \\
\hline & $\begin{array}{l}\text { Plantago major L. }\left(^{*}\right) \\
\text { Grand plantain /L-mesâsa }\end{array}$ & $\mathrm{Fe}$ & Cat & $\begin{array}{l}\text { ApL : Facilite le drainage de } \\
\text { l'abcès en cas de } \\
\text { furonculose. }\end{array}$ \\
\hline \multirow[t]{5}{*}{ Poaceae } & $\begin{array}{l}\text { Cynodon dactylon (L.) Pers. }\left(^{*}\right) \\
\text { Chiendent /Nnjem }\end{array}$ & TFe & Déc & Or : Contre le froid. \\
\hline & $\begin{array}{l}\text { Pennisetum typhoides (Burm.) } \\
\text { Stapf. \&Hubb. }\left(^{*}\right) \\
\text { Millet /îllân }\end{array}$ & $\mathrm{Gr}$ & $\mathrm{Ct}$ & Or: Facilite l'accouchement. \\
\hline & $\begin{array}{l}\text { Phragmites communis Trin. } \\
\text { Roseau /l-qseb }\end{array}$ & $\mathrm{Ra}$ & $\begin{array}{l}\text { PdrDHen en } \\
\text { Cat }\end{array}$ & ApC : Anti-chute. \\
\hline & $\begin{array}{l}\text { Triticum L. } \\
\text { Blé /Zr'aa / îrden }\end{array}$ & Sn & Déc & Or: Stomachique. \\
\hline & $\begin{array}{l}\text { Zeamays L. } \\
\text { Maïs /Dra }\end{array}$ & Stg & Déc & Or : Contre la constipation. \\
\hline \multirow[t]{3}{*}{ Ranunculaceae } & $\begin{array}{l}\text { Clematis flammula L. }\left(^{*}\right) \\
\text { Clématite flammette /Nâr el- } \\
\text { barda/ Azenzû }\end{array}$ & $\mathrm{Fe}$ & Cat & Or: Contre les verrues. \\
\hline & $\begin{array}{l}\text { Delphinium staphisagria L. }\left({ }^{*}\right) \\
\text { Dauphinelle staphysaigre } \\
\text { /Habbet-râs }\end{array}$ & $\mathrm{Gr}$ & Pdr & ApC : Antichute. \\
\hline & $\begin{array}{l}\text { Nigella sativa L. }\left(^{*}\right) \\
\text { Cumin noir /Sânûj }\end{array}$ & $\mathrm{Gr}$ & $\begin{array}{l}\text { PdrDM ou } \\
\text { PdrDH }\end{array}$ & $\begin{array}{l}\text { Or: Stomachique. Contre le } \\
\text { refroidissement, les maladies } \\
\text { respiratoires, et l'impuissance } \\
\text { sexuelle. }\end{array}$ \\
\hline \multirow[t]{2}{*}{ Rhamnaceae } & $\begin{array}{l}\text { Rhamnus alaternus L. } \\
\text { Nerprun alaterne /Melilez }\end{array}$ & TFe & Déc & Or : Contre l'anémia. \\
\hline & $\begin{array}{l}\text { Ziziphus lotus (L.) Lam. } \\
\text { jujubier sauvage } \\
\text { /Sedra/Nnbeg/Tazouggouart }\end{array}$ & $\mathbf{R a}$ & Pdr & $\begin{array}{l}\text { ApL: Contre les brûlures et } \\
\text { les blessures. Astringente. }\end{array}$ \\
\hline \multirow[t]{5}{*}{ Rosaceae } & $\begin{array}{l}\text { Crataegus monogyna Jacq. } \\
\text { Aubépine monogyne/Za'arour/ } \\
\text { Admâm }\end{array}$ & TFe & Déc & $\begin{array}{l}\text { Or: Anti-diarrhéique. } \\
\text { Hypotenseur. }\end{array}$ \\
\hline & $\begin{array}{l}\text { Crataegus laciniata Ucria } \\
\text { Aubépine /Tabgha }\end{array}$ & $\mathrm{Fe}$ & Déc & Or: Stomachique. \\
\hline & $\begin{array}{l}\text { Malus domestica Borkh. } \\
\text { Pommier commun /Teffâh }\end{array}$ & $\mathrm{Fr}$ & Jus & Or : Anti-diabétique. \\
\hline & $\begin{array}{l}\text { Prunus dulcis (Mill.) D.A.Webb } \\
\text { Amandier /Lûz }\end{array}$ & $\mathrm{Gr}$ & $\begin{array}{l}\text { PdrDHen } \\
\text { en Cat }\end{array}$ & ApC : Assouplissant. \\
\hline & Pyrus communis $\mathrm{L}$. & $\mathrm{Fr}$ & Jus & Or: Contre l'impuissance \\
\hline
\end{tabular}


Benlamdini et al. J. Appl. Biosci. 2014. Étude floristique et ethnobotanique de la flore médicinal du Haute Moulouya, Maroc

\begin{tabular}{|c|c|c|c|c|}
\hline & Poirier /Bû-'wid & & & sexuelle. \\
\hline & $\begin{array}{l}\text { Rosa canina L. } \\
\text { Eglantier /Ward /Tighfert }\end{array}$ & $\mathrm{Fe}$ & $\begin{array}{l}\text { Déc } \\
\text { Pdr }\end{array}$ & $\begin{array}{l}\text { Or: Stomachique. Contre la } \\
\text { dysfonction érectile. } \\
\text { ApT/ApC : Contre le maux de } \\
\text { tête. Antichute. }\end{array}$ \\
\hline Rubiaceae & $\begin{array}{l}\text { Rubia tinctorum L. } \\
\text { Garance des teinturiers/Fuwa/ } \\
\text { Tarûbya }\end{array}$ & $\mathrm{Ra}$ & Déc & Or: Stomachique. \\
\hline \multirow[t]{2}{*}{ Rutaceae } & $\begin{array}{l}\text { Citrus limon (L.) Burm.f. } \\
\text { Citronnier /El Hâmmed }\end{array}$ & $\mathrm{Fr}$ & Jus/MélDM & $\begin{array}{l}\text { Or : Contre les maladies } \\
\text { respiratoires et les maladies } \\
\text { cardiaques. }\end{array}$ \\
\hline & $\begin{array}{l}\left.\text { Ruta montana (L.) L. ( }{ }^{*}\right) \\
\text { Ru sauvage /Fîjel / âwermi }\end{array}$ & $\mathrm{Ra}$ & Déc & Or: Contre l'anémie. \\
\hline Salicaceae & $\begin{array}{l}\text { Salix alba L. } \\
\text { Saule Vivier /Ud el-mâ }\end{array}$ & $\mathrm{Fe}$ & Déc & Or: Antidiabétique. \\
\hline Schisandraceae & $\begin{array}{l}\text { Illicium verum Hook. f. } \\
\text { Anis étoilé /Badiane }\end{array}$ & $\mathrm{Fe}$ & PdrDM & Or: Contre l'asthme. \\
\hline Solanaceae & $\begin{array}{l}\text { Datura stramonium L. }\left({ }^{*}\right) \\
\text { Datura officinal /Sdeqej-jmel / } \\
\text { Taburzigt }\end{array}$ & $\mathrm{Fe}$ & $\mathrm{Cr}$ & $\begin{array}{l}\text { Inh: Somnifères pour les } \\
\text { enfants. }\end{array}$ \\
\hline \multirow[t]{2}{*}{ Thymelaeaceae } & $\begin{array}{l}\text { Daphne gnidium L. }\left(^{*}\right) \\
\text { Garou /Lezzâz }\end{array}$ & $\mathrm{Fe}$ & $\begin{array}{l}\text { PdrDHen en } \\
\text { Cat }\end{array}$ & ApL : Antichute. Anti-galeux. \\
\hline & $\begin{array}{l}\text { Daphne laureola L. }\left(^{*}\right) \\
\text { Daphné lauréole /Talzzâzt }\end{array}$ & $\mathrm{Fe}$ & $\begin{array}{l}\text { PdrDHen en } \\
\text { Cat }\end{array}$ & ApL : Antichute. Anti-galeux. \\
\hline Urticaceae & $\begin{array}{l}\text { Urtica pilulifera } \mathrm{L} . \\
\text { Ortie romaine /l-hurryqa }\end{array}$ & $\mathrm{Fe}$ & Déc /MélDH & $\begin{array}{l}\text { ApL: Contre les eczémas } \\
\text { des pieds. }\end{array}$ \\
\hline Verbenaceae & $\begin{array}{l}\text { Aloysia citrodora Palau } \\
\text { Verveine odorante /Lwîza }\end{array}$ & $\mathrm{Fe}$ & Inf ou Mac & $\begin{array}{l}\text { Or: Contre l'insomnie, et les } \\
\text { douleurs abdominales. } \\
\text { Hypotenseur. Rafraîchissant. }\end{array}$ \\
\hline Vitaceae & $\begin{array}{l}\text { Vitis vinifera L. } \\
\text { Vigne /La'anb }\end{array}$ & $\mathrm{Fe}$ & Pdr en Cat & ApT : Fébrifuge. \\
\hline Xanthorrhoeaceae & $\begin{array}{l}\text { Aloe succotrina All. }\left(^{*}\right) \\
\text { Aloès /Sebar /Sibra }\end{array}$ & HE & $\mathrm{Cr}$ & ApC : Assouplissant. \\
\hline \multirow[t]{3}{*}{ Zingiberaceae } & $\begin{array}{l}\text { Alpinia officinarum Hance } \\
\text { Galangal officinal /Hôdenjal }\end{array}$ & $\begin{array}{l}\mathrm{Rh} \\
\mathrm{Rh}\end{array}$ & $\begin{array}{l}\text { Déc } \\
\text { Pdr }\end{array}$ & $\begin{array}{l}\text { Or: Stomachique. Fébrifuge. } \\
\text { Contre le froid et la grippe. } \\
\text { UE : Contre les brulures. }\end{array}$ \\
\hline & $\begin{array}{l}\text { Elettaria cardamomum (L.) } \\
\text { Maton } \\
\text { Cardamome /Qa'qolla }\end{array}$ & $\mathrm{Gr}$ & Déc & $\begin{array}{l}\text { Or: Contre le maux des } \\
\text { dents. }\end{array}$ \\
\hline & $\begin{array}{l}\text { Zingiber officinalis Rosc. } \\
\text { Gingembre /Zanjabile /Skenjbir }\end{array}$ & Rh & Cat & $\begin{array}{l}\text { ApLDs: Contre le } \\
\text { refroidissement. }\end{array}$ \\
\hline Zygophyllaceae & $\begin{array}{l}\text { Zygophyllum gaetulum Emb \& } \\
\text { Maire } \\
\text { Zygophylle /Lâagaya }\end{array}$ & $\mathrm{Fe}$ & Déc & $\begin{array}{l}\text { Or: Contre les affections } \\
\text { urinaires. }\end{array}$ \\
\hline
\end{tabular}

$(*)$ : Plante toxique. (l) : Et.

Partie utilisée

$\mathrm{Bb}$ : Bulbe, Cls : Clous, Ec : Ecorce, EcDFr : Ecorce de Fruits, Fe : Feuille, Fr : fruit, Gld : Glands, Gr : Grains, HE : Huile Essentielle, Nx : Noix, PA : Partie Aérienne, Ra : racine, Rh : Rhizome, SFI : Sommités Fleuries, Sn : Son, Stg : stigmates, $\mathbf{T}$ : Tige, TFe : Tige Feuillée, Tgm : Téguments, TgmDNx : Téguments de Noix. 
Benlamdini et al. J. Appl. Biosci. 2014. Étude floristique et ethnobotanique de la flore médicinal du Haute Moulouya, Maroc

Mode de préparation

Cat : Cataplasme, $\mathrm{Cr}$ : $\mathrm{Cru}, \mathrm{CrDH}:$ Cru dans huile, $\mathrm{Ct}$ : Cuit, CtDH : Cuit dans huile, Déc : Décoction, Fum : Fumigation, Inf : Infusion, Jus : Jus, Mac : Macération, Mas : Massage, MélDH : Mélange dans huile, MélDM : Mélange dans miel, Pdr : Poudre, PdrDH : Poudre dans huile, PdrDHen : Poudre dans henné, PdrDL : Poudre dans lait, PdrDM : Poudre dans miel.

Mode d'administration

ApC : Application sur le cheveu, ApCCC : Application sur le cheveu et sur le cuir chevelu, ApL : Application locale, ApLDs : Application sur le dos, ApT : Application sur la tête, BaBo : Bain de bouche, BaPds : Bain de pieds, Col : Collyre, Me : Dans mets, Inh : Inhalation, Or : Orale, UE : Usage externe. 